\title{
Evaluación de la adherencia a las recomendaciones para disminuir el riesgo de Síndrome de Muerte Súbita del Lactante.
}

\author{
MANUEL ROCCA R. ${ }^{1}$, JUAN BOSCH F. ${ }^{1}$, CATERINA HENSON D. ${ }^{1}$, \\ PABLO REYES H. ${ }^{1}$, MICAELA CONDE A. ${ }^{2}$, MILAGROS RISSO R. ${ }^{2}$, \\ NÉSTOR ANTONIO GÓMEZ P. ${ }^{3}$, PABLO ATCHABAHIAN C. ${ }^{4}$ \\ 1. Departamento Materno Infantil, Hospital Universitario Austral, Argentina. \\ 2. Facultad de Ciencias Biomédicas, Universidad Austral, Argentina. \\ 3. Hospital Municipal Comodoro Meisner, Pilar, Argentina. \\ 4. Secretaría de Salud del Partido de Pilar, Argentina.
}

\begin{abstract}
Adherence to recommendations to reduce the risk of Sudden Infant Death Syndrome

Introduction: Recommendations for Sudden Infant Death Syndrome (SIDS) are available, although it is uncertain the degree of adherence to these measures. The aim of the study is to assess the adherence to recommendations to reduce the risk of SIDS, seeking factors associated to the noncompliance with these recommendations. Patients and Method: 468 infants were enrolled in two maternity hospitals, one public and one private. Postpartum and 4-month assessments were performed. A questionnaire was used adapting a model validated by the International Child Care Practices Study. Results: Adherence to obstetric recommendations was higher $(75.4 \%)$ than to pediatric recommendations $(53.3 \%)$. Regarding pediatric recommendations, a compliance decrease was detected after 4 months. Follow-ups showed decrease in breastfeeding $(\mathrm{p}<0.001$ $84.9 \%$ vs $48.6 \%)$ and supine position $(61.2 \%$ vs $21.2 \%, \mathrm{p}<0.001)$. Conclusions: The decreased adherence to recommendations for SIDS prevention was mainly observed in younger and less educated women, who were not in a relationship and living in poor housing quality and crowded environments.

(Key words: Sudden infant death syndrome, adherence, risk factors).

Rev Chil Pediatr 2014; 85 (4): 462-469
\end{abstract}

\section{RESUMEN}

Introducción: Existen recomendaciones preventivas en el Sindrome de Muerte Súbita del lactante (SMSL), aunque es incierto el grado de adherencia a dichas medidas. El objetivo del estudio fue evaluar la adherencia a las recomendaciones para disminuir el riesgo de SMSL, buscando factores asociados al no cumplimiento de

Recibido el 07 de julio de 2013, última versión aceptada para publicación el 19 de marzo de 2014.

Potenciales conflictos de interés: Este trabajo cumple con los requisitos sobre consentimiento /asentimiento informado, comité de ética, financiamiento, estudios animales y sobre la ausencia de conflictos de intereses según corresponda.

Correspondencia a:

Juan Bosch F.

E-mail: drjjbosch@gmail.com 
dichas recomendaciones. Pacientes y Método: Se incluyeron 468 recién nacidos de dos maternidades, pública y privada. Se efectuó una evaluación posparto y a los 4 meses de la adherencia a las recomendaciones. Se utilizó un cuestionario adaptando un modelo de encuesta validado por el International Child Care Practices Study Resultados: La adherencia a las recomendaciones obstétricas fue mayor $(75,4 \%)$ que a las recomendaciones pediátricas $(53,3 \%)$. A su vez en las recomendaciones pediátricas se evidenció una caída del cumplimiento de las recomendaciones a los 4 meses. Al seguimiento se observó reducción de lactancia $(84,9 \%$ vs 48,6\%; $\mathrm{p}<0,001)$ y de posición supina $(61,2 \%$ vs $21,2 \% ; \mathrm{p}<0,001)$. Conclusiones: La disminución de la adherencia a las recomendaciones para la prevención del SMSL se observó principalmente en las mujeres de menor edad y menor nivel educativo, no estar en pareja, mala calidad habitacional y presencia de numerosos convivientes. (Palabras clave: Síndrome de muerte súbita del lactante, adherencia, factores de riesgo).

Rev Chil Pediatr 2014; 85 (4): 462-469

\section{Introducción}

El síndrome de muerte súbita del lactante (SMSL) representa un importante problema de salud no sólo por constituir una de las principales causas de mortalidad post neonatal en muchos países, incluyendo la Argentina, sino también por lo trágico, inesperado y hasta ahora inexplicable del proceso. El niño aparentemente sano antes de acostarlo en su cuna aparece muerto horas más tarde.

Esto comprende parte de la definición que establece al síndrome como la muerte repentina e inesperada de un niño menor de un año de edad que permanece sin explicación después de una investigación completa del caso, incluida la realización de una autopsia completa, el examen de la escena de la muerte, y la revisión de la historia clínica ${ }^{1-3}$.

Hacia la década de 1990, en numerosos países desarrollados se implementaron campañas públicas con el fin de educar a la población sobre los factores de riesgo y hábitos de crianza asociados SMSL, entre ellos la posición prona al dormir, logrando en promedio una reducción del 53\% de la incidencia del SMSL y del $39 \%$ de la mortalidad post neonatal ${ }^{3}$.

En Argentina, se han desarrollado estrategias similares de difusión a partir del año 1997, organizadas por entidades científicas como la Sociedad Argentina de Pediatría, organismos gubernamentales y no gubernamentales.

Actualmente puede considerarse que las últimas recomendaciones elaboradas por el Grupo de Trabajo en SMSL de la Academia
Americana de Pediatría $^{3}$ constituyen las de mayor aceptación y grado de evidencia científica, reconociéndose que el nivel A incluye medidas asociadas a un beneficio sustancial y difícilmente modificables por futuros estudios (tabla 1$)^{3}$.

La implementación de estas recomendaciones a nivel de la población general reduce significativamente la tasa de prevalencia del $\mathrm{SMSL}^{4}$. Sin embargo, son muy escasos los datos de seguimiento sobre la adherencia a estas medidas en latinoamerica. El objetivo principal del estudio es evaluar la adherencia a las recomendaciones de prevención del SMSL. El objetivo secundario es evaluar factores asociados al no cumplimiento de las recomendaciones.

\section{Pacientes y Método}

\section{Diseño}

Estudio prospectivo, descriptivo y multicéntrico en una amplia población representativa del $90 \%$ de los nacimientos del Partido de Pilar, Buenos Aires. El diseño del estudio incluyó un trabajo de campo recolectando información a partir de encuestas y entrevistas en el Hospital Universitario Austral (HUA) y el Hospital Municipal Comodoro Meisner (Hospital Meisner en adelante). Se tomó una muestra de 321 pacientes (170 del HUA y 151 del Hospital Meisner) que representó un nivel de confianza del $98,5 \%(\alpha=0,015)$ al considerar una prevalencia de la posición prona al dormir del $30 \%{ }^{4-6}$. 
Tabla 1. Recomendaciones de nivel A de la AAP* para un medio ambiente seguro del lactante al dormir

1. Posición para dormir siempre en decúbito dorsal
2. Usar una superficie firme de apoyo al dormir
3. Cohabitación pero sin compartir la cama
4. Mantener objetos blandos y ropa de cama suelta alejados de la cuna
5. Cuidados prenatales adecuados y regulares
6. Evitar la exposición al tabaco durante la gestación y el período posnatal
7. Evitar el consumo de alcohol y de drogas ilícitas durante la gestación y el período posnatal
8. Alimentación a través del pecho materno
9. Considerar el uso de chupete durante el sueño
10. Evitar el sobrecalentamiento
11. No use monitores cardiorrespiratorios como una estrategia para reducir el riesgo de SMSL
12. Expanda la campaña nacional para reducir el riesgo de SMSL para incluir un mayor enfoque en el ambiente de sueño seguro y
formas de reducir los riesgos de toda muerte infantil relacionada con el sueño, incluyendo SMSL, asfixia y otras muertes acciden-
tales; pediatras, médicos de familia y otros profesionales de atención primaria deben participar activamente en esta campaña

*Task Force on Sudden Infant Death Syndrome, Moon RY. SIDS and other sleep-related infant deaths: expansion of recommendations for a safe infant sleeping environment. Pediatrics 2011; 128 (5): 1030-9.

Se realizaron dos evaluaciones: a) adquisición de datos basales correspondiente a los meses de noviembre-diciembre de 2011, y b) resultados al seguimiento (4 meses después) correspondiente a abril-mayo de 2012.

Fueron incluidos recién nacidos vivos $>$ a 35 semanas de gestación. Los criterios de exclusión fueron los siguientes: Malformaciones congénitas mayores e internación en unidad de cuidados intensivos neonatales $(\mathrm{UCIN})>10$ días.

\section{Aspectos éticos}

El estudio fue aprobado por el CIE (Comité Institucional de Evaluación) de la Facultad de Ciencias Biomédicas de la Universidad Austral. Todas las madres, padres o tutores aceptaron y firmaron el consentimiento informado escrito respectivo.

\section{Recolección de datos}

Los datos fueron recolectados en ambos centros administrando un cuestionario ad hoc durante la internación previo al alta (basal) $\mathrm{y}$ otro posterior al nacimiento (seguimiento) $4 \pm 0,5$ meses de vida.

Todo el personal incluido en el estudio realizó una inducción de cómo sería el estudio.
Se formaron grupos focales donde se explicó cada procedimiento. Las personas participantes realizaron la misma capacitación sobre el llenado de las encuestas previo al inicio del estudio con el investigador principal del estudio. Se realizaron tres capacitaciones de refuerzo a lo largo de la duración del estudio.

$\mathrm{Si}$ bien en la instancia basal la recolección fue enteramente presencial, al seguimiento se concretaron entrevistas telefónicas o personales, de acuerdo con las limitaciones de la familia y los requerimientos del caso particular.

El cuestionario se configuró adaptando el modelo de encuesta validado, realizado por el International Child Care Practices Study ${ }^{6-8}$. Consta de una etapa basal realizada en el puerperio inmediato y un cuestionario de seguimiento realizado a los 4 meses de vida.

\section{Análisis estadístico}

Con el programa SPSS versión 15.0 para Windows se efectuó un análisis descriptivo, con prueba de chi-cuadrado para las variables dicotómicas. Se efectuó MANCOVA (las variables de filiación de la madre y de antecedentes socioeconómicos como covariables) y ANOVA univariados para identificar los factores de riesgo de las anteriores recomendaciones. 
Tabla 2. Características de las madres y cumplimiento de las recomendaciones obstétricas

\begin{tabular}{|c|c|c|c|}
\hline \multicolumn{2}{|c|}{ Características de la madre } & $\begin{array}{r}n \\
6\end{array}$ & $\begin{array}{l}\text { \% } \\
1,9\end{array}$ \\
\hline Edad (años) & $\begin{array}{c}<15 \\
15-19 \\
20-29 \\
30-39 \\
>40\end{array}$ & $\begin{array}{r}6 \\
42 \\
145 \\
122 \\
6\end{array}$ & $\begin{array}{r}1,9 \\
13,1 \\
45,2 \\
38,0 \\
1,9\end{array}$ \\
\hline $\begin{array}{l}\text { Nivel de } \\
\text { instrucción }\end{array}$ & $\begin{array}{l}\text { Terciario/Universitario } \\
\text { Secundario completo } \\
\text { Secundario incompleto } \\
\text { Primario completo } \\
\text { Primario incompleto } \\
\text { Analfabeta }\end{array}$ & $\begin{array}{r}84 \\
93 \\
50 \\
56 \\
36 \\
2\end{array}$ & $\begin{array}{r}26,2 \\
29,0 \\
15,6 \\
17,4 \\
11,2 \\
0,6\end{array}$ \\
\hline Estado civil & $\begin{array}{l}\text { En pareja } \\
\text { Solteras } \\
\text { Separada }\end{array}$ & $\begin{array}{r}284 \\
36 \\
1\end{array}$ & $\begin{array}{r}88,5 \\
11,2 \\
0,3\end{array}$ \\
\hline $\begin{array}{l}\text { n de emba- } \\
\text { razos }\end{array}$ & $\begin{array}{l}<3 \\
3 \text { a } 5 \\
>5\end{array}$ & $\begin{array}{r}204 \\
88 \\
29\end{array}$ & $\begin{array}{r}63,6 \\
27,4 \\
9,0\end{array}$ \\
\hline n de hijos & $\begin{array}{l}<3 \\
3 \text { a } 5 \\
>5\end{array}$ & $\begin{array}{r}220 \\
77 \\
24\end{array}$ & $\begin{array}{r}68,5 \\
24,0 \\
7,5\end{array}$ \\
\hline $\begin{array}{l}\mathrm{n} \text { de convi- } \\
\text { vientes }\end{array}$ & $\begin{array}{l}2 \\
3 \\
4 \\
5 \\
>5\end{array}$ & $\begin{array}{r}4 \\
83 \\
92 \\
66 \\
76\end{array}$ & $\begin{array}{r}1,2 \\
25,9 \\
28,7 \\
20,6 \\
23,7\end{array}$ \\
\hline Recomendac & iones obstétricas & & \\
\hline $\mathrm{n}$ de controles & $\begin{array}{l}<3 \\
3 \text { a } 5 \\
>5\end{array}$ & $\begin{array}{r}28 \\
98 \\
195\end{array}$ & $\begin{array}{r}8,7 \\
30,5 \\
60,7\end{array}$ \\
\hline $\begin{array}{l}\text { Tabaquismo } \\
\text { materno }\end{array}$ & $\begin{array}{l}\text { No } \\
<5 \text { cigarrillos/d } \\
5-10 \text { cigarrillos/d } \\
>10 \text { cigarrillos/d }\end{array}$ & $\begin{array}{r}277 \\
31 \\
10 \\
3\end{array}$ & $\begin{array}{r}86,3 \\
9,7 \\
3,1 \\
0,9\end{array}$ \\
\hline $\begin{array}{l}\text { Ingesta de } \\
\text { alcohol }\end{array}$ & $\begin{array}{l}\text { No } \\
\text { Sí }\end{array}$ & $\begin{array}{r}254 \\
67\end{array}$ & $\begin{array}{l}79,1 \\
20,9\end{array}$ \\
\hline
\end{tabular}

\section{Resultados}

\section{Población}

Fueron incluidos en el estudio 468 madres y $\mathrm{RN}$ que cumplían los criterios de inclusión. La pérdida de datos al seguimiento (4 meses) de los $468 \mathrm{RN}$ incluidos inicialmente, fue de 147 participantes $(31,4 \%)$ debido en mayor medida a la inexistencia del número telefónico registrado, o su inhabilitación: El total de la muestra en seguimiento a los 4 meses fue de 321 participantes (170 del HUA y 151 del Hospital Meisner).

\section{Evaluacion inicial}

Fueron características principales de la población basal, madres de 20-34 años, con al menos secundario completo, en pareja, con vivienda de material, menos de 3 embarazos e hijos, y $\mathrm{RN}$ de 3.001-4.000 $\mathrm{g}$ al nacer, parto a término $\mathrm{y}$ vigorosos (tabla 2).

\section{Seguimiento}

Evidenciamos un mayor nivel de adherencia a las recomendaciones obstétricas (tabla 3), el $60,7 \%$ realizó más de 5 controles del embarazo, el $86,3 \%$ no fumó durante el embarazo y el $79,1 \%$ no ingirió alcohol durante embarazo, con un cumplimiento global del $75,36 \%$. En relación a las recomendaciones pediátricas (figura 1) evidenciamos una disminución en las variables a los 4 meses del nacimiento. La lactancia materna bajó del $84,9 \%$ al $48,6 \%$, el uso de chupete del 53,8\% al 50,2\% y el dormir en posición supina del $61,2 \%$ al $21,2 \%$, siendo que a los 4 meses el 50,3\% de los niños dormía en posición de costado.

Tabla 3. Relación entre las características de la población y las recomendaciones preventivas sobre el SMSL

\begin{tabular}{|c|c|c|c|c|c|c|}
\hline \multirow{6}{*}{ 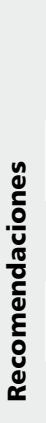 } & & \multicolumn{5}{|c|}{ Variables } \\
\hline & & $\begin{array}{l}\text { Edad de la } \\
\text { madre }\end{array}$ & $\begin{array}{l}\text { Nivel de instrucción } \\
\text { de la madre }\end{array}$ & $\begin{array}{l}\text { Tipo de } \\
\text { vivienda }\end{array}$ & $\begin{array}{l}\text { Estado civil } \\
\text { de la madre }\end{array}$ & $\begin{array}{l}\text { Cantidad de } \\
\text { convivientes }\end{array}$ \\
\hline & $\begin{array}{l}\text { Más de } 5 \text { controles } \\
\text { durante el embarazo }\end{array}$ & $\begin{array}{l}\text { Madre } \geq 25 \\
\text { años }\end{array}$ & $\begin{array}{l}\text { A partir del secundario } \\
\text { completo }\end{array}$ & $\begin{array}{l}\text { Mejor calidad de } \\
\text { vivienda (material) }\end{array}$ & $\begin{array}{l}\text { Presencia de } \\
\text { pareja }\end{array}$ & $\begin{array}{l}\text { No se observó } \\
\text { relación }\end{array}$ \\
\hline & $\begin{array}{l}\text { Posición supina de } \\
\text { sueño }\end{array}$ & $\begin{array}{l}\text { Madre } \geq 20 \\
\text { años }\end{array}$ & $\begin{array}{l}\text { A partir de primario } \\
\text { completo }\end{array}$ & $\begin{array}{l}\text { No se observó } \\
\text { relación }\end{array}$ & $\begin{array}{l}\text { Presencia de } \\
\text { pareja }\end{array}$ & $\begin{array}{l}\text { No se observó } \\
\text { relación }\end{array}$ \\
\hline & Uso de chupete & $\begin{array}{l}\text { No se observó } \\
\text { relación }\end{array}$ & $\begin{array}{l}\text { A partir de primario } \\
\text { completo }\end{array}$ & $\begin{array}{l}\text { No se observó } \\
\text { relación }\end{array}$ & $\begin{array}{l}\text { Presencia de } \\
\text { pareja }\end{array}$ & $\begin{array}{l}\text { No se observó } \\
\text { relación }\end{array}$ \\
\hline & $\begin{array}{l}\text { Duerme en cuna o } \\
\text { moisés }\end{array}$ & $\begin{array}{l}\text { No se observó } \\
\text { relación }\end{array}$ & No se observó relación & $\begin{array}{c}\text { Vivienda de material } \\
\text { o casillas }\end{array}$ & $\begin{array}{l}\text { No se observó } \\
\text { relación }\end{array}$ & $\geq 3$ convivientes \\
\hline
\end{tabular}




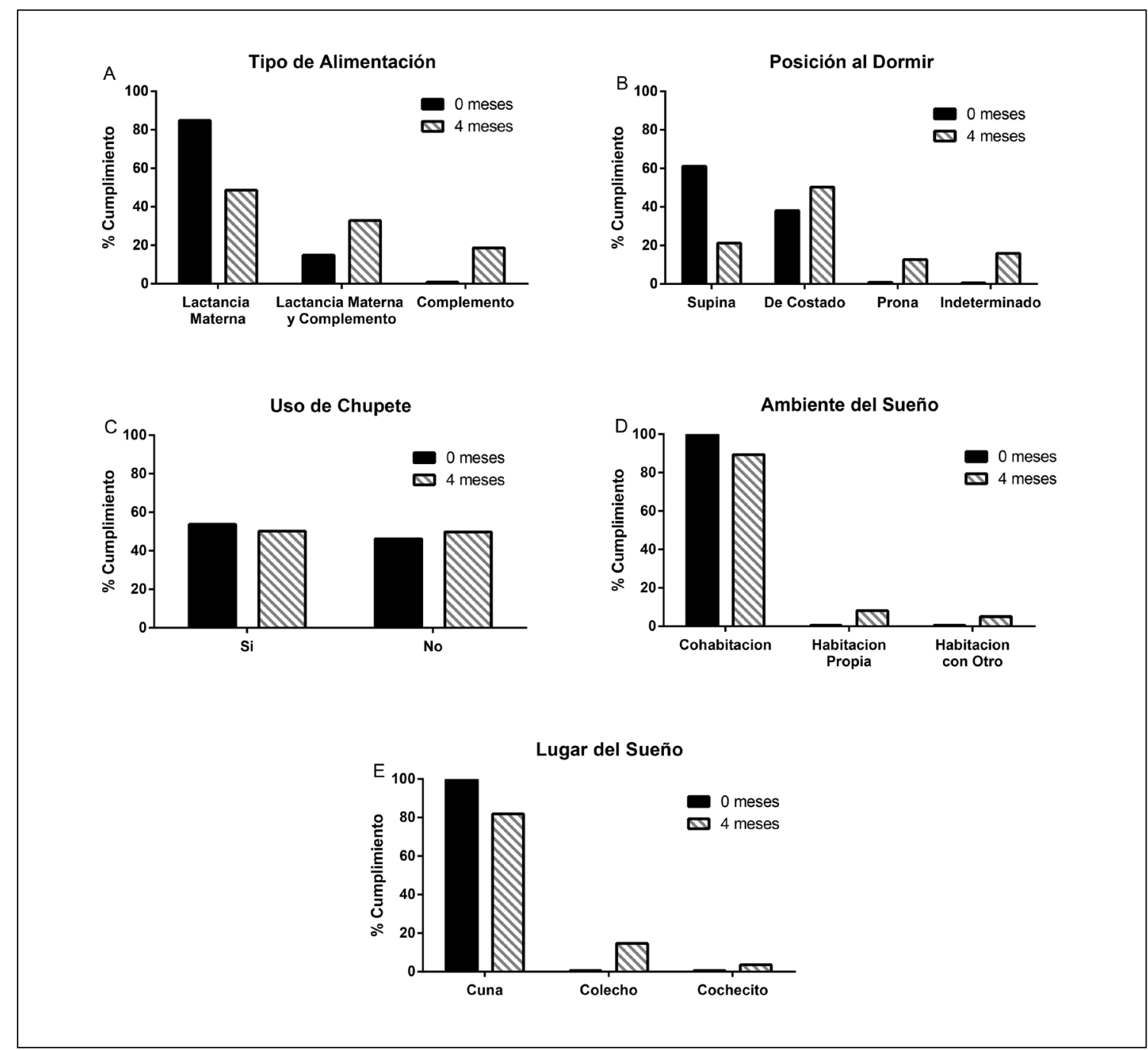

Figura 1. Cumplimiento de las recomendaciones pediátricas preventivas del SMSL.

\section{Discusión}

De acuerdo con Blair et $\mathrm{al}^{9}$, la mayoría de los casos de SMSL ocurren actualmente en familias con bajos niveles socioeconómicos y educativos, grupo que presenta más dificultad para el cumplimiento de las medidas preventivas del SMSL ${ }^{10}$.

Mientras que en numerosos países desarrollados como EE.UU, Noruega, Nueva Zelanda, Canadá y Reino Unido la prevalencia de la posición supina al dormir es mayor al $70 \%$ a los 4 meses de vida ${ }^{9-14}$, en nuestra población fue 3 veces menor, prevaleciendo la posición de costado, con una baja incidencia de la posición prona al dormir.

Existió un cambio significativo de la posición al dormir desde el alta hospitalaria posparto al control a los 4 meses de edad (de posición supina hacia la posición de costado), datos concordantes con los de Lesko et al ${ }^{15}$, en este caso asociado a la población de origen hispano, madres jóvenes ( $<25$ años) y con bajos niveles de educación. La posición al dormir continua a la fecha siendo un hábito difícil de modificar, existen temores respecto de que la 
posición en decúbito supino sea insegura, que aumente la posibilidad de aspiraciones ${ }^{16}$ y que genere un mayor número de despertares ${ }^{17}$.

Se encuentran bien establecidos los efectos del tabaquismo sobre la salud perinatal y posnatal, incluyendo principalmente un bajo peso al nacer, parto pre término y un riesgo aumentado para el SMSL ${ }^{18-23}$. Las campañas de prevención sobre el SMSL en los países desarrollados influyeron muy poco sobre el hábito de fumar en las madres. En el Reino Unido este incluso se incrementó entre las madres de niños con SMSL (del 50\% al 80\%), cuando dicha tasa se redujo entre las embarazadas de la población general (del 30\% al 20\%) ${ }^{18}$. Los porcentajes de mujeres que fuman durante el embarazo hallados en el presente estudio fueron similares a los encontrados en trabajos previos efectuados en hospitales públicos de la Argentina $(11 \%)^{24}$. Los datos hallados en este estudio evidencian un serio problema de salud materna y perinatal, siendo aún más preocupante al considerar las cifras de exposición ambiental al tabaco ( 1 cada 4 mujeres convive con fumadores).

Situación más compleja se evidencia al evaluar el consumo de alcohol durante el embarazo. Las diversas sociedades científicas nacionales e internacionales indican que las mujeres embarazadas no deben ingerir ninguna forma del mismo, de lo contrario incrementan los riesgos de problemas fetales del alcohol. El consumo de alcohol es un factor de riesgo para el SMSL, aunque no está claro si interfiere independientemente o en combinación con otros como el cigarrillo ${ }^{16}$. En nuestra población cerca de 1 cada 4 cada mujeres indicó ingerir alcohol durante el embarazo, esto asociándose con aquellas que fumaron $>10$ cigarrillos/día.

La lactancia materna (LM) es reconocida como la forma óptima de alimentación temprana dados sus innumerables beneficios sobre la salud materna y de los niños, tanto a corto como a largo plazo. En su estudio, Hauck et $\mathrm{al}^{25}$ concluyeron que la LM exclusiva protege contra el SMSL. Los resultados del presente trabajo concordaron con aquellos hallados por el Ministerio de Salud en la Encuesta de Lactancia Materna $2007^{26}$, en este último se hallaron valores a los 4 meses de edad del $46 \%$ para la LM exclusiva, del 33\% para la LM parcial y del 14\% para el destete.

Sobre el uso de chupete para dormir existe una fuerte asociación entre su utilización y la disminución del riesgo del $\mathrm{SMSL}^{25}$. En el caso de los niños amamantados debe ofrecerse el chupete cuando el niño ya ha cumplido el primer mes de vida, para así asegurar que la lactancia esté bien establecida. En nuestro estudio, el uso de chupete representó uno de los factores de peor cumplimiento.

En relación al ambiente de la vivienda y el lugar preciso donde duerme el $\mathrm{RN}$, ambas recomendaciones fueron ampliamente cumplidas (cohabitación con los padres y cuna/ moisés, respectivamente), el segundo parámetro no concordando con lo observado en el meta análisis de Vennemann et $\mathrm{al}^{27}$, en tanto el hallazgo de casi 3 veces mayor en la proporción de colecho.

Parece razonable que los esfuerzos destinados a reducir los riesgos del SMSL deben apuntar a los grupos de población que corren un riesgo especial. Por ejemplo, ante el caso de la posición prona al dormir, las estrategias de atención prenatal y/o neonatal parecen ser suficientes al nacimiento, pero no para los 4 meses de vida del niño. Siendo esta la edad de mayor prevalencia, se requiere de diferentes estrategias de intervención para disminuir el riesgo de SMSL. La consulta en control de niño sano puede ofrecer una oportunidad adicional para discutir la posición de sueño como un factor de riesgo para el SMSL.

Por su parte, la recomendación de amamantamiento debe incluirse en las campañas de reducción del riesgo de SMSL y en relación con muchos otros beneficios de salud del niño. Los organismos internacionales recomiendan el amamantamiento exclusivo hasta los 6 meses de vida y que, a partir de ese momento, se comience a introducir alimentos sin abandonar la $\mathrm{LM}^{26}$.

Observamos ciertas características asociadas al no cumplimiento de las recomendaciones preventivas: edad, nivel de instrucción y estado civil de la madre, tipo de vivienda y cantidad de convivientes. Si bien estas, fueron específicas sobre una u otra recomendación, resulta esencial considerarlas conjuntamente 
para así diseñar e implementar con mayor eficacia cualquier estrategia preventiva sobre el SMSL a nivel poblacional.

Finalmente, se reconoce como limitación del estudio el estar circunscrito en una sola región de la Provincia de Buenos Aires, hecho atenuado al incluir el $90 \%$ de los partos en el Partido de Pilar. Su poder de extrapolación a otras regiones del país dependerá de la comparación de las características socioeconómicas y culturales. También es una debilidad del estudio la pérdida en el seguimiento de pacientes, en el cuestionario de seguimiento a los 4 meses, ya que podría constituir un sesgo de evaluación.

En conclusión, en el seguimiento de los pacientes a los 4 meses hemos evidenciado una caída significativa en el cumplimiento de las recomendaciones pediátricas de prevención del SMSL. Esta caída se asoció en mayor medida a menor edad y nivel educativo de la madre, el no estar en pareja, una mala calidad habitacional y la presencia de numerosos convivientes. La determinación de los niveles de adherencia (y sus factores de riesgo) sobre las recomendaciones preventivas del SMSL podría ser útil para aportar datos representativos del estado de prevención en la población evaluada, lo que además facilitaría el establecimiento de recomendaciones de mayor riesgo de incumplimiento. Esto último podría constituir la base para la identificación de aquellas situaciones en las que se requieran mejores estrategias de difusión de las medidas de prevención. Se sugiere el diseño e implementación de campañas preventivas públicas orientadas a la población en general, pero no descartando intervenciones sobre subpoblaciones de características específicas.

\section{Agradecimientos}

Al Departamento de Desarrollo Académico del Hospital Universitario Austral por la ayuda brindada en el área estadística y de corrección del trabajo, a las Dras. Belén Quesada, María José Negri Aranguren, Paola Vaccaro y Ana María López por su asesoría en la recolección de datos.

\section{Referencias}

1.- Willinger M, James $L S$, Catz C: Defining the sudden infant death syndrome (SIDS): deliberations of an expert panel convened by the National Institute of Child Health and Human Development.Pediatr Pathol 1991; 11: 677-84.

2.- American Academy of Pediatrics AAP Task Force on Infant Positioning and SIDS: Positioning and SIDS. Pediatrics 1992; 89 (6 Pt 1): 1120-6.

3.- Task Force on Sudden Infant Death Syndrome, Moon $R Y$ : SIDS and other sleep-related infant deaths: expansion of recommendations for a safe infant sleeping environment. Pediatrics 2011; 128 (5): 1030-9.

4.- Eymann A, Ricciardi M, Caprotta G, Fustiñana C, Jenik $A$ : Cambios en la posición al dormir para la prevención de la muerte súbita del lactante: diez años de seguimiento. An Pediatr (Barc) 2008; 68 (3): 244-9.

5.- Rocca Rivarola M, Burgos F, General F, Fernández I: Evaluación de los hábitos de crianza en niños menores de 6 meses en el partido de Pilar. 2do Congreso Argentino de Pediatría General Ambulatoria y Encuentro de Sociedades de Pediatría del Cono Sur; 2001 Nov 15-18; Buenos Aires, Argentina: SAP.

6.- Cafferata ML, Althabe F, Belizán JM, et al; Grupo de Estudio sobre Consejos en las Maternidades (MAS Study group) para América Latina y el Caribe: Posición al dormir en hospitales de América Latina y el Caribe para la prevención del síndrome de muerte súbita del lactante. An Pediatr (Barc) 2002; 57 (6): 558-64.

7.- Nunes ML, Martins MP, Nelson EA, Cowan S, Cafferata $M L$, Costa JC: Instructions from teaching hospital maternity wards to parents concerning the sleeping position of newborns. Cad Saude Publica 2002; 18 (3): 883-6.

8.- Nelson EA, Serra A, Cowan S, Mangiaterra V: Maternity advice survey: sleeping position in Eastern Europe. MAS Study Group for WHO EURO region. Arch Dis Child 2000; 83 (4): 304-6.

9.- Blair PS, Sidebotham P, Berry PJ, et al: Major epidemiological changes in sudden infant death syndrome: a 20-year population-based study in the UK. Lancet 2006; 367 (9507): 314-9.

10.- Pickett KE, Luo Y, Lauderdale DS: Widening social inequalities in risk for sudden infant death syndrome. Am J Public Health 2005; 95 (11): 1976-81.

11.- Scragg RK, Mitchell EA: Side sleeping position and bed sharing in the sudden infant death syndrome. Ann Med 1998; 30 (4): 345-9.

12.- Mitchell EA, Tonkin S: Publicity and infants' sleeping position. BMJ 1993; 306 (6881): 858. 
13.- Hiley CM, Morley CJ: Evaluation of government's campaign to reduce risk of cot death. BMJ 1994; 309 (6956): 703-4.

14.- Kahn A, Groswasser J, Sottiaux M, et al: Prone or supine body position and sleep characteristics in infants. Pediatrics 1993; 91 (6): 1112-5.

15.- Lesko SM, Corwin MJ, Vezina RM, et al: Changes in sleep position during infancy: a prospective longitudinal assessment. JAMA 1998; 280 (4): 336-40.

16.- Krous HF, Masoumi H, Haas EA, et al: Aspiration of gastric contents in sudden infant death syndrome without cardiopulmonary resuscitation. J Pediatr 2007; 150 (3): 241-6.

17.- Franco P, Groswasser J, Sottiaux M, et al: Decreased cardiac responses to auditory stimulation during prone sleep. Pediatrics 1996; 97 (2): 174-8.

18.- McDonnell-Naughton M, McGarvey C, O'Regan M, Matthews T: Maternal smoking and alcohol consumption during pregnancy as risk factors for sudden infant death. Ir Med J 2012; 105 (4): 105-8.

19.- Liebrechts-Akkerman G, Lao O, Liu F, et al: Postnatal parental smoking: an important risk factor for SIDS. Eur J Pediatr 2011; 170 (10): 1281-91.

20.- Owen L, McNeill A, Callum C: Trends in smoking during pregnancy in England, 1992-7: quota sampling surveys. BMJ 1998; 317 (7160): 728-30.

21.- Fleming P, Blair PS: Sudden Infant Death Syndrome and parental smoking. Early Hum Dev 2007; 83 (11): 721-5.

22.- Franco P, Groswasser J, Hassid S, et al: Prenatal exposure to cigarette smoking is associated with a decrease in arousal in infants. J Pediatr 1999; 135 (1): 34-8.

23.- Kahn A, Groswasser J, Sottiaux M, et al: Prenatal exposure to cigarettes in infants with obstructive sleep apneas. Pediatrics 1994; 93 (5): 778-83.

24.- Althabe F, Colomar M, Gibbons L, et al: Tabaquismo durante el embarazo en Argentina y Uruguay. Medicina (Buenos Aires) 2008; 68 (1):48-54.

25.- Hauck FR, Thompson JM, Tanabe KO, et al: Breastfeeding and reduced risk of sudden infant death syndrome: a meta-analysis. Pediatrics 2011; 128 (1): 103-10.

26.- Ministerio de Salud: Situación de Lactancia Materna en Argentina. Año 2007. Buenos Aires, Argentina: Dirección Nacional de Maternidad e Infancia, Ministerio de Salud de la Nación, 2007.

27.- Vennemann MM, Hense HW, Bajanowski T, et al: Bed sharing and the risk of sudden infant death syndrome: can we resolve the debate? J Pediatr 2012; 160 (1): 44-8. 